\title{
ON FUNCTIONS HAVING NON-NEGATIVE DERIVATIVES AT THE ORIGIN ${ }^{1}$
}

R. G. LAHA

Functions which have non-negative derivatives at the origin play an important role in the theory of probability. The moment generating functions of symmetric distributions and the probability generating functions of lattice distributions are examples of such functions which are most commonly used. In the present note, we give two theorems on the properties of such functions. These theorems have interesting applications in the theory of decomposition of distribution functions which is known as "arithmetic of distributions."

First we give the following definition:

Definition. Let $f(x)$ be a function of the real variable $x$ which has finite derivatives of all orders

$$
f^{(k)}(0)=\left[\frac{d^{k}}{d x^{k}} f(x)\right]_{x=0}, \quad k=1,2,3, \cdots,
$$

at the origin $x=0$. We then define formally

$$
F(z)=f(0)+\sum_{k=1}^{\infty} \frac{f^{(k)}(0)}{k !} z^{k} \quad(z \text { complex })
$$

as a function of the complex variable $z(z=x+i y, x$ and $y$ both real) and call $F(z)$ the adjoint function of $f(x)$. In general, the function $F(z)$ may not exist except at the point $z=0$ and we note that $F(0)=f(0)$. The adjoint function $F(z)$ is said to be regular in a circle $|z|<R$ $(R>0)$ about the origin $z=0$ of the complex $z$-plane if and only if the power series $f(0)+\sum_{k=1}^{\infty}\left(f^{(k)}(0) / k !\right) z^{k}$ converges in the same circle $|z|<R$.

We are now in a position to formulate the following theorem:

Theorem 1. Let $f(x), f_{1}(x), f_{2}(x), \cdots, f_{n}(x)$ be $(n+1)$ functions of the real variable $x$ which have non-negative derivatives of all orders at the origin and let $\alpha_{1}, \alpha_{2}, \cdots, \alpha_{n}$ be $n$ positive numbers. Let the adjoint function $F(z)$ of $f(x)$ be regular in a circle $|z|<R(R>0)$ about the point $z=0$. Suppose that the functions $f_{j}(x)(j=1,2, \cdots, n)$ are positive and satisfy the relation

Received by the editors November 11, 1959 and, in revised form, February 26, 1960 and May 9, 1960.

1 This work was supported by the National Science Foundation through grant NSF-G-4220. 


$$
\left[f_{1}(x)\right]^{\alpha_{1}}\left[f_{2}(x)\right]^{\alpha_{2}} \cdots\left[f_{n}(x)\right]^{\alpha_{n}}=f(x)
$$

for all real $x$ in the interval $0 \leqq x \leqq \delta(\delta>0)$.

Then each of the adjoint functions $F_{j}(z)$ of $f_{j}(x)$ is also regular at least in the circle $|z|<R$.

This theorem has been proved by D. Dugue in [1]. For the sake of completeness we give below an alternative proof of this theorem. The method of proof is similar to the one used by $\mathrm{Yu}$. V. Linnik and A. A. Zinger in [2]. We first prove the following lemma:

LEMma. Under the conditions of Theorem 1 each of the adjoint functions $F_{j}(z)$ is regular in some circle about the point $z=0$.

Proof. First, we note that the function $[f(x)]^{N}$ (where $N$ is a positive integer) is also positive at the origin and has non-negative derivatives of all orders at the origin. It is also easy to verify that for any $k(k=1,2,3, \cdots)$

$$
\left[\frac{d^{k}}{d x^{k}}[f(x)]^{N}\right]_{x=0}=\left[\frac{d^{k}}{d z^{k}}[F(z)]^{N}\right]_{z=0} .
$$

We now raise both sides of (1) to a suitable positive integer power $p$ such that each $\beta_{j}=p \alpha_{j} \geqq 1$.

Next we again raise both sides of the resulting equation to a positive integer power $k$ and thus obtain

$$
\left[f_{1}(x)\right]^{k \beta_{1}}\left[f_{2}(x)\right]^{k \beta_{2}} \cdots\left[f_{n}(x)\right]^{k \beta_{n}}=[f(x)]^{k p} .
$$

We then differentiate both sides of (3) $k$ times with respect to $x$ and then put $x=0$. Finally we write the result in the form

$$
S_{1}(0)+S_{2}(0)=\left[\frac{d^{k}}{d x^{k}}[f(x)]^{k p}\right]_{x=0}
$$

where $S_{1}(0)$ contains only the derivatives of order $k$ of the functions $f_{j}(x)(j=1,2, \cdots, n)$, while $S_{2}(0)$ consists of all the derivatives of order not exceeding $k-1$. We can then verify easily that

$$
S_{1}(0)=[f(0)]^{k p} \sum_{j=1}^{n} k \beta_{j} \frac{f_{j}^{(k)}(0)}{f_{j}(0)}
$$

while each summand in $S_{2}(0)$ is non-negative so that $S_{2}(0) \geqq 0$. Thus we obtain from (2), (4) and (5) the inequality 


$$
\begin{aligned}
f_{j}^{(k)}(0) & \leqq \frac{1}{k \beta_{j}} \cdot \frac{f_{j}(0)}{[f(0)]^{k p}} \cdot\left[\frac{d^{k}}{d x^{k}}[f(x)]^{k p}\right]_{x=0} \\
& \leqq \frac{f_{j}(0)}{[f(0)]^{k p}} \cdot\left[\frac{d^{k}}{d z^{k}}[F(z)]^{k p}\right]_{z=0}
\end{aligned}
$$

holding for all $j=1,2, \cdots, n$ and $k=1,2, \cdots$. We shall now estimate the right-hand side of (6) with the help of Cauchy's integral (cf. $[3$, p. 82]). Since the function $F(z)$ is regular in the circle $|z|<R$ about the point $z=0$, we can write the right-hand side of (6) in the form

$$
\left[\frac{d^{k}}{d z^{k}}[F(z)]^{k p}\right]_{z=0}=\frac{k !}{2 \pi i} \oint_{|z|=R / 2} \frac{[F(z)]^{k p}}{z^{k+1}} d z .
$$

We set $M_{1}=\max _{|z|-R / 2}|F(z)|$. Then we see clearly that $M_{1}>0$ and we obtain from (7) the estimate

$$
\left|\left[\frac{d^{k}}{d z^{k}}[F(z)]^{k p}\right]_{z=0}\right| \leqq k ! \frac{M_{1}{ }^{k p}}{(R / 2)^{k}} .
$$

We then combine (6) and (8) and get finally

$$
f_{j}^{(k)}(0) \leqq f_{j}(0) \frac{k !}{(R / 2)^{k}} \cdot\left[M_{1} / f(0)\right]^{k p} .
$$

It follows at once from (9) that the power series

$$
f_{j}(0)+\sum_{k=1}^{\infty} f_{j}^{(k)}(0) \cdot \frac{z^{k}}{k !}
$$

converges in a circle about the point $z=0$ with a radius of convergence which is not less than

$$
\rho_{1}=\frac{R}{2} \cdot\left[f(0) / M_{1}\right]^{p}>0 .
$$

Therefore each of the adjoint functions $F_{j}(z)$ is regular at least in the circle $|z|<\rho_{1}$ about the origin. This completes the proof of the lemma.

We now turn to the proof of the theorem. We have to show that each of the adjoint functions $F_{j}(z)$ is regular in a circle about the origin with a radius of convergence not less than $R$. Let us suppose that this is not the case. Then at least one of the functions $F_{j}(z)$ converges in a circle about the origin with a radius which is less than $R$. Let $R_{0}$ be the smallest of the radii of convergence of all the functions $F_{j}(z)$. Then according to our assumption $R_{0}<R$ so that $\rho=R-R_{0}>0$. 
Since each of the functions $F_{j}(z)$ has non-negative derivatives of all orders at the origin, it follows at once from the theorem of Pringsheim and Landau (cf. [3, p. 214]) that the point of singularity of the function $F_{j}(z)$ which is nearest to the origin must be located on the positive half of the real axis. Therefore, the point $z=R_{0}$ must be a singular point of at least one of the functions $F_{j}(z)$ which is situated nearest to the point $z=0$. We further note that the relation

$$
\left[F_{1}(z)\right]^{k \beta_{1}}\left[F_{2}(z)\right]^{k \beta_{2}} \cdots\left[F_{n}(z)\right]^{k \beta_{n}}=[F(z)]^{k p}
$$

holds for complex values of $z$ in circle $|z|<R_{0}$ about the origin. We then write $\rho_{0}=R_{0}-\epsilon$ where $\epsilon\left(0<\epsilon<R_{0} / 10\right)$ is a small number. Then we substitute $x=\rho_{0}+\xi$ and introduce the functions

$$
\begin{aligned}
G_{j}(\xi) & =\frac{F_{j}\left(\rho_{0}+\xi\right)}{F_{j}\left(\rho_{0}\right)}, \quad j=1,2, \cdots, n, \\
G(\xi) & =\frac{F\left(\rho_{0}+\xi\right)}{F\left(\rho_{0}\right)} .
\end{aligned}
$$

First, we note that the functions $G_{j}(\xi)$ and $G(\xi)$ are also positive at the point $\xi=0$ and have non-negative derivatives of all orders at the point $\xi=0$. It is also easy to verify from (10) that the relation

$$
\left[G_{1}(\xi)\right]^{k \beta_{1}}\left[G_{2}(\xi)\right]^{k \beta_{2}} \cdots\left[G_{n}(\xi)\right]^{k \beta_{n}}=[G(\xi)]^{k p}
$$

holds for all real $\xi$ in the interval $0 \leqq \xi<\epsilon$. We also remark that the function $G(\zeta)$ of the complex variable $\zeta(\zeta=\xi+i \eta, \xi$ and $\eta$ are both real) is regular at least in the circle $|\zeta|<\rho$, where $\rho=R-R_{0}>0$. We repeat the same operation as above with the equation (11) and obtain the inequality

$$
G_{j}^{(k)}(0) \leqq \frac{G_{j}(0)}{[G(0)]^{k p}} \cdot\left[\frac{d^{k}}{d \zeta^{k}}[G(\zeta)]^{k p}\right]_{\zeta=0}
$$

holding for all $j=1,2, \cdots, n$ and $k=1,2, \cdots$. We then estimate the right-hand side of (12) with the help of Cauchy's integral. Thus we have

$$
\left[\frac{d^{k}}{d \zeta^{k}}[G(\zeta)]^{k p}\right]_{\zeta=0}=\frac{k !}{2 \pi i} \oint_{|\zeta|=\rho / 2} \frac{[G(\zeta)]^{k p}}{\zeta^{k+1}} d \zeta .
$$

We set $M_{2}=M_{2}(\rho)=\max _{|\zeta|=p / 2}|G(\zeta)|$ and thus obtain from (12) and (13) the estimate

$$
G_{j}^{(k)}(0) \leqq G_{j}(0) \cdot \frac{k !}{(\rho / 2)^{k}} \cdot\left[M_{2} / G(0)\right]^{k p}
$$


holding for all $j=1,2, \cdots, n$ and $k=1,2, \cdots$. Thus we conclude from (14) that for every $\epsilon$ in the interval $0<\epsilon<R_{0} / 10$, each of the functions $G_{j}(\zeta)$ converges in a circle about the point $\zeta=0$ with a radius of convergence which is not less than $\rho_{2}=(\rho / 2)\left[G(0) / M_{2}\right]^{p}>0$. We also note that $\rho_{2}$ is independent of $\epsilon$. But on the other hand the radius of the circle of convergence of at least one of the functions $G_{j}(\zeta)$ must be $\epsilon$. Thus we obtain the desired contradiction by selecting $\epsilon$ sufficiently small in the interval $0<\epsilon<R_{0} / 10$. This completes the proof of the theorem.

TheOREM 2. Under the same conditions as in Theorem 1, let the adjoint function $F(z)$ of $f(x)$ be an entire function of some finite order $\gamma$. Then each of the adjoint functions $F_{j}(z)$ of $f_{j}(x)$ is also an entire function of some finite order not exceeding $\gamma$.

Proof. It follows immediately from Theorem 1 that each $F_{j}(z)$ is an entire function whenever $F(z)$ is an entire function. Thus the relation

$$
\left[F_{1}(z)\right]^{\alpha_{1}}\left[F_{2}(z)\right]^{\alpha_{2}} \cdots\left[F_{n}(z)\right]^{\alpha_{n}}=F(z)
$$

holds for complex values of $z$. We now introduce the functions

$$
\begin{aligned}
H_{j}(z) & =F_{j}(z) / F_{j}(1), \quad j=1,2, \cdots, n, \\
H(z) & =F(z) / F(1),
\end{aligned}
$$

and verify easily from (15) that the functions $H_{j}(z)$ satisfy the relation

$$
\left\lceil H_{1}(R)\right]^{\alpha_{1}}\left[H_{2}(R)\right]^{\alpha_{2}} \cdots\left[H_{n}(R)\right]^{\alpha_{n}}=H(R)
$$

for all real $R$. We also note that for $R \geqq 1$

$$
H_{j}(R) \geqq 1, \quad j=1,2, \cdots, n,
$$

so that we obtain from (16) the inequality

$$
\left[H_{j}(R)\right]^{\alpha_{j}} \leqq H(R)
$$

holding for $R \geqq 1$ and $j=1,2, \cdots, n$. Let $M\left(R ; H_{j}\right)$ and $M(R ; H)$ denote the maximum modulus of the functions $H_{j}(z)$ and $H(z)$ respectively in the circle $|z| \leqq R$. This value is evidently attained on the perimeter of this circle and in view of the non-negativity of the derivatives at the origin we see easily that

$$
\begin{aligned}
M\left(R ; B_{j}\right) & =\max _{|z|=R}\left|H_{j}(z)\right|=H_{j}(R), \quad i=1,2, \cdots, n, \\
M(R ; H) & =\max _{|z|=R}|H(z)|=H(R) .
\end{aligned}
$$


Thus we get at once from (17) the inequality

$$
\left[M\left(R ; H_{j}\right)\right]^{\alpha_{j}} \leqq M(R ; H)
$$

holding for all $R \geqq 1$ and $j=1,2, \cdots, n$. We now note that

$$
\gamma=\limsup _{R \rightarrow \infty} \frac{\ln \ln M(R ; H)}{\ln R}
$$

so that the proof follows at once from (18) and (19).

Theorem 2 yields the following useful corollary.

Corollary. Let $f_{1}(x), f_{2}(x), \cdots, f_{n}(x)$ be $n$ functions which have non-negative derivatives of all orders at the origin and let $\alpha_{1}, \alpha_{2}, \cdots, \alpha_{n}$ be $n$ positive numbers. Suppose that the functions $f_{j}(x)$ are positive and satisfy the relation

$$
\left[f_{1}(x)\right]^{\alpha_{1}}\left[f_{2}(x)\right]^{\alpha_{2}} \cdots\left[f_{n}(x)\right]^{\alpha_{n}}=\exp [P(x)]
$$

for all real $x$ in the interval $0 \leqq x \leqq \delta(\delta>0)$ where $P(x)$ is a polynomial in $x$ of degree $m$ such that $\exp [P(x)]$ has a non-negative derivative at the origin. Then each of the adjoint functions $F_{j}(z)$ of $f_{j}(x)$ has the form $F_{j}(z)=\exp \left[P_{j}(z)\right]$ where $P_{j}(z)$ is a polynomial in $z$ of degree not exceeding $m$.

Proof. From Theorems 1 and 2, it follows at once that each of the adjoint functions $F_{j}(z)$ is an entire function of finite order not exceeding $m$ and further the relation

$$
\left[F_{1}(z)\right]^{\alpha_{1}}\left[F_{2}(z)\right]^{\alpha_{2}} \cdots\left[F_{n}(z)\right]^{\alpha_{n}}=\exp [P(z)]
$$

holds for complex values of $z$. We see easily from (20) that none of the functions $F_{j}(z)$ can have any zeros in the entire complex plane. The proof is then an immediate consequence of the factorization theorem of Hadamard (cf. [3, p. 250]). It is interesting to note that Theorem 1.2 in [1] is a special case of this result.

\section{REFERENCES}

1. D. Dugué, Sur le thêrème de Levy-Cramer, Publ. Inst. Statist. Univ. Paris vol. 6 (1958) pp. 213-225.

2. Yu. V. Linnik and A. A. Zinger, On an analytical extension of a theorem of Cramér and its application (in Russian), Vestnik. Leningrad. Univ. vol. 10 (1955) pp. 51-56.

3. E. C. Titchmarsh, The theory of functions, Oxford, University Press, 1939.

Catholic University of America 